\title{
A Comparison of Psychiatrist Evaluation and Patient Symptom Report in a Jail Telepsychiatry Clinic
}

\author{
EVE-LYNN NELSON, Ph.D., ${ }^{1}$ CHARLES ZAYLOR, M.D., ${ }^{2}$ and DAVID COOK, Ph.D. ${ }^{3}$
}

\begin{abstract}
The effectiveness of a jail telepsychiatry service was evaluated by comparing psychiatrist and inmate report of psychopathology. Sixty-two inmates completed a total of 107 consultations at a rural county jail via interactive televideo. The inmates completed the Symptom Rating Checklist-90-Revised (SCL-90-R), and the psychiatrist completed a Psychiatrist Evaluation Form including the Clinical Global Impression Scale-Severity Index (CGI) after each teleconsultation. Most inmates were rated mild to moderately ill on the CGI. There was a significant, high correlation between telepsychiatrist evaluation on the CGI and inmate report of overall symptoms on the SCL-90-R $[r(101)=0.35, p<0.05]$. The findings support the effectiveness of telepsychiatric evaluation for the jail population. The patterns of telepsychiatric use in the county jail as well as future directions in this setting are described.
\end{abstract}

\section{INTRODUCTION}

$\mathbf{R}$ URAL CORRECTIONAL FACILITIES face a difficult balance in providing mental health services to inmates. On the one hand, there is substantial need within the correctional setting for mental health services. Individuals in the correctional setting have a higher incidence of mental illness than the general population, in addition to history of substance abuse. ${ }^{1}$ They also may experience adjustment reactions to the correctional setting such as difficulty in sleeping, anxiety, and depression. In addition to ethical-legal considerations, untreated inmates are also more likely to engage in fights and rule violations. ${ }^{1}$ On the other hand, the nation in general and rural areas in particular face severe shortages in mental health resources. For example, 96 of 105 counties in Kansas are designated Mental Health Professional Shortage areas. Even when care is available in the county, few providers have experience or training in correctional psychiatry.

Correctional facilities are interested in telepsychiatric services as a solution to shortages, in addition to other telemedicine applications within the correctional setting. ${ }^{2}$ When available, face-to-face psychiatry still carries the cost of transport and the risk of escape. Psychiatry has offered services using technology for over 40 years, ${ }^{3}$ with videoconferencing applications increasing rapidly over the last few years. ${ }^{4}$ While patients report satisfaction with telepsychiatric services, ${ }^{5}$ providers are also concerned about establishing the effectiveness of diagnosis and treatment in the new context. The majority of literature in telepsychiatry includes accounts of novel clinical demonstra-

\footnotetext{
${ }^{1}$ Center for Telemedicine and Telehealth and ${ }^{3}$ Health \& Technology Outreach, Kansas University Medical Center, Kansas City, Kansas.

${ }^{2}$ Lansing Correctional Facility, Lansing, Kansas.
} 
tions and descriptions of program projects, with a handful of empirical evaluations. ${ }^{6}$

Several studies have compared psychiatric evaluation of the same patient face-to-face and over telemedicine $\mathrm{e}^{7-11}$ and have found high agreement between face-to-face and telemedicine. No such comparison has been reported in the jail setting. Questions related to the clinician's ability to assess symptoms as well as clinical effectiveness in this setting are important as more and more correctional facilities adopt telepsychiatric interventions. The current study addresses agreement between psychiatric evaluation over telemedicine and inmate self-report of symptoms. Previous reports ${ }^{12}$ describe symptom improvement from both the psychiatrist and inmate perspectives over time, while the current study compares psychiatrist and inmate ratings in terms of global and specific measures of psychological functioning. It addresses the following research questions:

- Research Question 1 (RQ1): What are the diagnostic characteristics of inmates presenting in a rural jail telemedicine clinic?

- Research Question 2 (RQ2): How do overall psychiatric ratings compare with overall patient ratings of functioning?

- Research Question 3 (RQ3): How does psychiatric evaluation of depressive symptoms compare with inmate self-report of depressive symptoms?

\section{MATERIALS AND METHODS}

\section{Background}

Because of the provider shortages and the high need for mental health services, the University of Kansas Center for TeleMedicine and TeleHealth (KUCTT) was approached by the National Law Enforcement and Corrections Technology Advisory Council-Southeast and its partners (the Joint Program Steering Group, the National Institute of Justice Office of Science and Technology, and the Federal Bureau of Prisons). Their goal was to assist correctional facilities in providing telepsychiatric services. KUCTT solicited participation in the project in jails across the state. A contract with a rural county jail was negotiated as a pilot for rural jail telepsychiatry. KUCTT and the county jail shared costs in the startup.

KUCTT contacted the psychiatrist in the Kansas University Medical Center (KUMC) Department of Psychiatry and Behavioral Sciences and coordinated the program. Through meeting with the county jail, KUCTT and the KUMC Department of Psychiatry established the jail's telepsychiatry needs and developed a protocol for implementing services. ${ }^{13}$

The county jail is a modern facility with approximately 140 beds. It is staffed by approximately 20 officers and houses both male and female inmates. Some individuals remain in jails for extended periods of time, but most are short-term residents awaiting either temporary or final release or movement to prisons to serve out their sentence. The facility also rents space to urban jails faced with overcrowding, and at times, up to half the jail population is from outside the county.

The jail used to acquire psychiatric consultations from the local mental health center, from the local hospital, and from a distant provider who traveled over 100 miles to the jail. The psychiatrist could only visit the jail at set times, approximately $1 / 2$ day per month. Thus, telemedicine offered increased access to care for inmates. This provided for early intervention with mild psychopathology and increased follow-up opportunity, as well as solving concerns around transporting inmates to the mental health center and to the hospital in a cost-effective manner.

The telepsychiatrist provides the consultation on a fee-for-service contract with the jail. This was more cost-effective than paying for a distant provider to drive to the jail. The cost of the equipment was paid for through a grant from the Department of Justice, and the jail pays the long distance telephone charges. KUCTT and the jail shared other administrative costs, such as the setup and maintenance of the equipment.

The jail medical officers screen inmates for psychiatric consultation and schedule the sessions. Correctional officers attend the inmate's assessment and follow-up appointments. The psychiatrist provides both emergency consultation for inmates on suicide watch and long-term care for inmates with mental illness. The clinic began in August 1998 as a monthly clinic and as 
needed. The jail clinic was established with the expectation of five to 10 patients per month, predominately for crisis situations such as suicide watch. As the clinic evolved, the psychiatrist conducted approximately 71 consultations per month, with most patients seen for chronic rather than emergency care. There were no technical difficulties during the initial consultations. The clinic use patterns followed a quadratic trend (Fig. 1), indicating a higher rate of use of the telepsychiatric services over time. Reasons for the increase included: increased referral of new patients, follow-up care for inmates, and re-evaluation in times of crisis. The increase over time reflects the development of a positive working relationship between the psychiatrist and the jail staff as well as an awareness concerning appropriate referral. From November 1999 to May 2000, 62 inmates completed the Symptom Rating Checklist-90-Revised (SCL-90-R) before 107 separate consults. Half of the inmates were under 30 years of age. Most (91\%) were male and Caucasian $(84 \%)$. The psychiatric completed the psychiatrist evaluation form, including Clinical Global Impression Scale-Severity Index (CGI) rating, immediately after each consult.

\section{Equipment}

Both the jail and the Medical Center use PCbased videoconferencing systems. The desktop units transmit digitized images and data over integrated services digital network (ISDN) lines (128 kbps). The psychiatrist's unit is conveniently located in his office. The jail designated a room specifically for telemedicine services, which allows both the inmate and medical officer to be viewed on the screen, and is equipped with a blue background to enhance the video image. A fax machine is used to transmit patient information.

\section{Instruments}

SCL-90-R. The SCL-90-R ${ }^{14}$ self-report symptom inventory was completed every 2 weeks by the inmates. Each item is rated on a 5-point scale of distress. It contains nine primary symptom scales (Somatization, Obsessive-Compulsive, Interpersonal Sensitivity, Depression, Anxiety, Hostility, Phobic Anxiety, Paranoid Ideation, and Psychoticism). The scale of interest in the current study was the Depression scale. It reflects the range of the manifestations of clinical depression symptoms including dysphoric mood, withdrawal from activities, lack of motivation, and loss of energy. In addition, it addresses feelings of hopelessness, thoughts of suicide, and other cognitive and somatic correlates of depression. The SCL-90-R total score is a measure of current psychological status. The SCL-90-R takes approximately $15 \mathrm{~min}$ to complete. It has adequate internal consistency, test-retest reliability, and validity. ${ }^{15}$

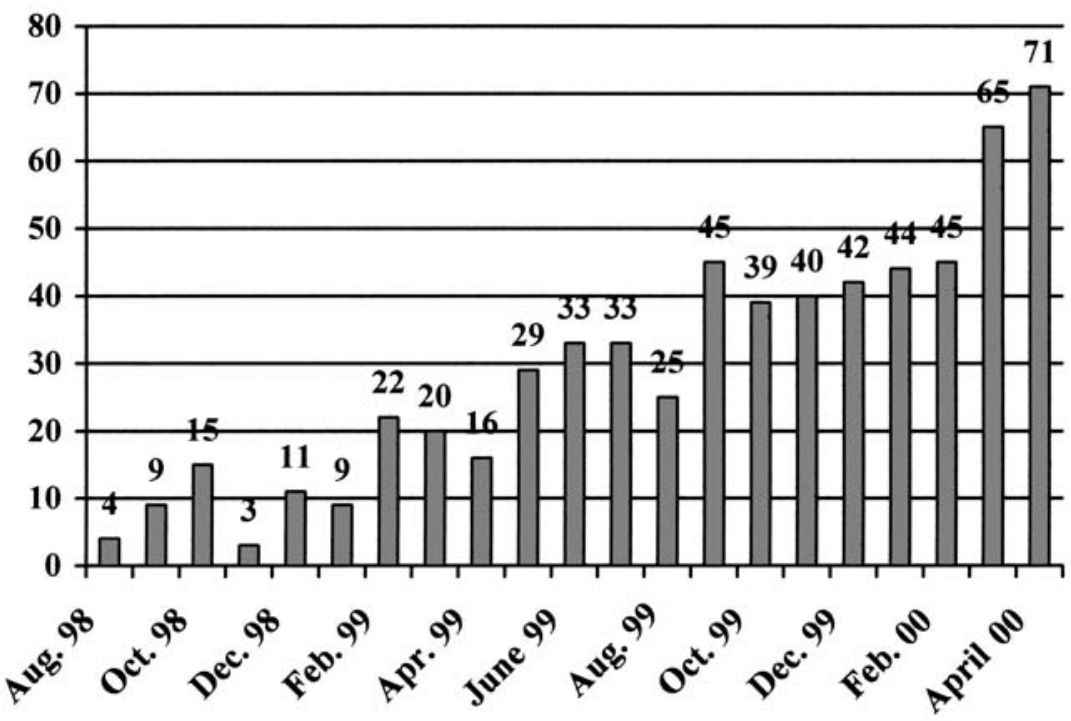

FIG. 1. Number of telepsychiatry consultations per month. 
Psychiatrist evaluation form. The psychiatrist completed a standard evaluation sheet following each consult. This included: patient history; a checklist concerning inmate mental status, affect, motor, speech, thoughts, hallucinations, delusions, suicidality, homicidality, and memory; treatment plan; and Clinical Global Impression Scale. The checklist component included the presence or absence of symptoms (e.g., has/does not have hallucinations) as well as descriptors (e.g., mood ratings included a checklist of constricted, euphoric, or dysphoric).

CGI. The CGI was completed by the psychiatrist at each consultation as part of the evaluation. It is a clinician-rated scale from 1 ("not at all ill") to 7 ("among the most extremely ill patients") that rates severity. This measure is used in psychopharmacological trials to measure symptom change. ${ }^{16}$

\section{Protocol}

Before meeting with the psychiatrist, the inmates completed the SCL-90-R, which was administered by the medical officer at the jail and mailed to the psychiatrist. During each session, the psychiatrist completed a Psychiatrist Evaluation Form.

\section{RESULTS}

The results will be reported according to each research question. To address the first question (RQ1), Table 1 presents the psychiatrist's impressions from the Psychiatrist Evaluation Form for the 107 consults. The most common diagnoses were mood disorders (44\%) and adjustment reactions (22\%). The mean CGI rating was $2.60(\mathrm{SD}=1.36)$, with the CGI frequencies presented in Table 2.

In relation to the second question (RQ2), a Pearson correlation coefficient was computed between the CGI and SCL-90-R total score. The correlation was significant: $r(101)=0.35, p<$ 0.05 .

To address the third question (RQ3), planned comparisons were run to compare specific psychiatrist ratings with inmate self-report. A Bon-
Table 1. Symptoms at First Tele-Psychiatric CONSUltation

Symptoms at first

psychiatric consultation

$(\mathrm{n}=62)$

Percentage

Affect

Constricted 33

Dysphoric $\quad 22$

Euthymic 63

Motor

Agitated

Hyper

Thoughts

Goal directed

Tangential

Delusion

Hallucinations

Suicidal

Homicidal

Memory

Remote intact 100

Recent intact $\quad 100$

Immediate intact 100

Medication prescribed at first visit $\quad 88$

ferroni procedure was used to control for Type II error across multiple comparisons, yielding a significant $p$ value of $0.01(0.05 / 4)$.

An independent samples $t$ test was conducted to evaluate the hypothesis that the psychiatrist's rating of suicidality would correlate with inmate self-report of thoughts of death. The SCL-90-R thoughts of death item was higher in inmates rated suicidal by the psychiatrist. The $t$ test was significant: $t(103)=-6.95$, $p<0.001 \quad($ mean $=1.24, \mathrm{SD}=1.57) \quad($ mean $=$ $3.50, \mathrm{SD}=0.58)$. The psychiatrist accurately identified all four inmates who expressed frequent suicidal ideation on the SCL-90R item.

Two independent samples $t$ tests were conducted to evaluate the hypothesis that the psychiatrist's rating of dysthymia and hyper-

Table 2. CGI Scale per Consultation

\begin{tabular}{lc}
\hline CGI per consultation & Number \\
\hline 1 Normal, not at all ill & 33 \\
2 Borderline ill & 10 \\
3 Mildly ill & 32 \\
4 Moderately ill & 18 \\
5 Markedly ill & 6 \\
6 Severely ill & 2 \\
7 Among the most extremely ill patients & 0 \\
None recorded & 6 \\
\hline
\end{tabular}


activity/restlessness would predict inmate self-report of depressive symptoms. The SCL90-R Depression scale was not significantly higher in inmates rated dysthmic by the psychiatrist $($ mean $=24.28, \mathrm{SD}=13.88)($ mean $=$ 28.87, SD = 14.77). The SCL-90-R Depression scale was significantly higher in inmates rated hyperactive/restless by the psychiatrist: $t(104)=-3.11, p<0.009($ mean $=24.74, \mathrm{SD}=$ 14.41) $($ mean $=32.86, \mathrm{SD}=5.73)$.

\section{DISCUSSION}

The discussion will outline five of the most critical "lessons learned" in the particular telemedicine project.

1. The rapid rate of increase in clinic utilization over a short time underscores the need to prepare for clinic volume before initiating a telehealth clinic. The increasing rate of telepsychiatry consults over 20 months was welcomed but not anticipated in initial planning. It reflects the medical officer's comfort in using the technology, his or her increased proficiency at identifying inmates appropriate for telepsychiatry consultation, and the clinic's transition from emergency care to care of inmates with chronic mental illness as well as emergency care. The high utilization (up to 70 consults per month) underscores a need for psychiatric service in rural jail populations. It also points to the likely cost-effectiveness of telejail services. In general the more consults, the more cost-effective is the telehealth consultation. Increased utilization offsets the startup costs.

2. For this rural jail population, the psychiatrist rated inmates as mild to moderately ill. This suggests inmates benefit from psychiatric services not only in crisis situations, but also in addressing more chronic mental illness such as the affective disorders, which can be effectively managed through telepsychiatry. These data suggest that more severe reactions are atypical even in a jail setting. Affective symptoms such as constricted and dysthymic mood were not common, nor were motor symptoms such as agitation or hyperactivity. Severe symptoms, such as hallucinations, delusions, suicidal thoughts, homicidal thoughts, and memory impairment, were rare.

3. The psychiatrist and inmate global ratings highly correlated with each other. Because the high correlation between the psychiatrist rating and inmate rating the psychiatrist was assured of the accuracy of assessing psychopathology over telemedicine. This supports reimbursement for telepsychiatric services.

4. Psychiatrist and inmate agreement concerning ratings of depressive symptoms depended on the type of item. The psychiatrist accurately identified all inmates who expressed frequent suicidal ideation. This is important as psychiatrists are asked to sit on involuntary medication hearings and other evaluations.

There was less concordance between inmate self-reported depressive symptoms and psychiatrist ratings of mood and motor activity on the Psychiatrist Evaluation Form. Inmates' self-report of depression was based on the multiple-item Depression scale of the SCL-90-R. The psychiatrist's ratings of depressive symptoms were two separate items on the Psychiatrist Evaluation Form: the assessment of mood item and the assessment of activity level item. In this sample, inmates' scores on the SCL-90-R Depression scale were significantly related to psychiatrist's ratings of motor activity, but not the psychiatrist's rating of dysthymic mood. It is difficult to determine whether this is a general challenge in diagnosis or if it relates to telemedicine. The psychiatrist's assessment of the activity level was more predictive than the psychiatrist's assessment of affect. This finding may also have been related to the relatively small sample size and to the low bandwidth utilized.

5. Future research needs to address the consequence of telepsychiatry services on the overall jail environment. The medical officers report that the service not only helps alleviate inmates' symptoms (affect distur- 
bance, sleep difficulty, etc.), but also leads to decreased inmate-inmate and inmatestaff violence.

\section{IMPLICATIONS}

This study supports the validity of psychiatric evaluation over telemedicine on the basis of the overlap between the psychiatrist's assessment and the inmate's self-report on global measures of psychiatric functioning. Nonetheless, the study had several limitations. One limitation is the reliance on a single instrument rather than a battery of validated self-report measures. Future studies may use a variety of methods (self-report, interview, and observation) as well as more specific questionnaires such as the Beck Depression Inventory to assess affect. Such evaluation will address concerns that the inmate population is not always truthful in self-presentation. A second limitation is use of a single provider. As pilot data support the expansion of telepsychiatric services, multi-site trials may be possible across several providers.

The study provides evidence that correctional telepsychiatry clinics would be well utilized by jails especially as jail officials become more accustomed to the process, just as has been suggested in the prison arena. ${ }^{17,18}$

The value of telepsychiatry services for inmates reaches beyond incarceration. The psychiatrist and jail are also considering how inmates may continue to receive care after their release.

\section{REFERENCES}

1. Ditton PM. Mental health and treatment of inmates and probationers. Special Report NCJ 174463. Washington, DC: Bureau of Justice Statistics, 1999.

2. Ellis DG, Mayrose J, Jehle DV, Moscati DM, Pierluisi GJ. A telemedicine model for emergency care in a short-term correctional facility. Telemed $J \mathrm{e}$-Health 2001;7:87-92.

3. Baer L, Elford R, Cukor P. Telepsychiatry at forty: what have we learned? Harv Rev Psychiatry 1997; 5:7-17.

4. Dahlin MP, Watcher G, Engle WM, Henderson J. Report on US telemedicine activity. Portland, OR: ATSP, 2001.
5. Mair F, Whitten P. Systematic review of studies of patient satisfaction with telemedicine. BMJ 2000;320: 1517-1520.

6. Frueh BC, Deitsch SE, Santos AB, Gold PB, Johnson MR, Meisler N, Magruder KM, Ballenger JC. Procedural and methodological issues in telepsychiatry research and program development. Psychiatr Serv 2000; 51:1522-1527.

7. Baigent MF, Lloyd CJ, Kavanagh SJ. Telepsychiatry: 'tele'yes, but what about the 'psychiatry'? J Telemed Telecare 1997;3:3-5.

8. Baer L, Cukor P, Jenike MA, Leahy L, O'Laughlen J, Coyle JT. Pilot studies of telemedicine for patients with obsessive-compulsive disorder. Am J Psychiatry 1995;152:1383-1385.

9. Elford DR, White H, St. John K, Maddigan B, Ghandi M, Bowering R. A prospective satisfaction study and cost analysis of a pilot child telepsychiatry service in Newfoundland. J Telemed Telecare 2001;7:73-81.

10. Ruskin PE, Reed S, Kumar R, Kling MA, Siegel E, Rosen M, Hauser P. Reliability and acceptability of psychiatric diagnosis via telecommunication and audiovisual technology. Psychiatr Serv 1998;49:1086-1088.

11. Zarate CA, Weinstock L, Cukor P, Morabito C, Leahy L, Burns C, Baer L. Applicability of telemedicine for assessing patients with schizophrenia: acceptance and reliability. J Clin Psychiatry 1997;58:22-25.

12. Zaylor C, Nelson E, Cook D. Telepsychiatry in a rural jail population. J Telemed Telecare 2001;7(Suppl 1):47-49.

13. Zaylor C, Whitten P, Kingsley C. Telemedicine services to a county jail. J Telemed Telecare 2000;6(Suppl 1): S93-S95.

14. Derogatis LR. SCL-90-R administration, scoring, and procedures manual-II. Towson, MD: Clinical Psychometric Research, Inc., 1992.

15. Ogles BM, Lambert MJ, Masters KS. Assessing outcome in clinical practice. Needham Heights, MA: Allyn \& Bacon, 1996.

16. Guy W. ECDEU assessment manual for psychopharmacology, revised. Rockville, MD: U.S. Department of Health and Human Services, 1976.

17. Zollo S, Kienkle M, Loeffelholz P, Sebille S. Telemedicine to Iowa's correctional facilities: initial clinical experience and assessment of program costs. Telemed J 1999;5:291-301.

18. Zincone LH, Doty E, Balch DC. Financial analysis of telemedicine in a prison system. Telemed J 1997;3:247255.

Address reprint requests to: Eve-Lynn Nelson, Ph.D. Center for Telemedicine and Telehealth Kansas University Medical Center Mail Stop 1048, 2012 Wahl Annex 3901 Rainbow Kansas City, KS 66160 E-mail: enelson2@kumc.edu 